\title{
Pressure Measurement in XHV Region Using Nonresonant Multiphoton Ionization by Picosecond Pulsed Laser
}

\author{
Kiyohide KOKUBUN, Shigeyuki SEKINE, Shingo ICHIMURA, \\ Akira KUROKAWA and Hazime SHIMIZU \\ Electrotechnical Laboratory, 1-1-4, Umezono, Tsukuba-shi, Ibaraki 305, Japan
}

(Received April 20, 1994, Accepted July 2, 1994)

By means of nonresonant multiphoton ionization using a picosecond pulsed YAG laser, pressure measurement for $\mathrm{H}_{2}, \mathrm{CO}$ and $\mathrm{CO}_{2}$ gases was carried out in the XHV pressure range. It was experimentally verified that pressure measurement was possible in the range of $10^{-11} \mathrm{~Pa}$, and this pressure measurement method had pressure sensitivity of the same order of magnitude for the three molecules.

\section{Introduction}

A great obstacle to the pressure measurement in the XHV region by means of nonresonant multiphoton ionization using lasers has been noise. In the case of excimer lasers, the scattered laser light produces noise. ${ }^{1)}$ Although this noise is significantly large, it can be removed by properly designing an ion-detection head. $\left.{ }^{2}\right)$ However, other noise of unknown origin still remains. For this reason, it had until recently possible only to measure pressures to $7 \times 10^{-10} \mathrm{~Pa}$ by means of the excimer laser.2)

Then, a picosecond pulsed laser was used for the purpose of increasing the ionization probability of molecules. As expected, saturation of ionization was realized for $\mathrm{H}_{2}, \mathrm{CO}, \mathrm{CO}_{2}$, and $\mathrm{Xe}$ by means of nonresonant multiphoton ionization. ${ }^{3,4)}$ In addition, use of the picosecond pulsed laser was found to bring an unexpected advantage in that the noise due to scattered laser light was not generated.5) This fact enabled us to use an ion-detection head with a much simpler structure, compared with that in the case of excimer lasers.

In the early stage of the experiment, however, noise similar to that in the excimer laser still remained. After some experiments, it was found that the noise seemed to result from photonstimulated desorption of ions at the view ports of the vacuum chamber, and could almost be removed through laser irradiation for about one hour at a repetition frequency of $10 \mathrm{~Hz}$. As a result, pressure measurements in the $10^{-11} \mathrm{~Pa}$ range for $\mathrm{H}_{2}$, and in the $10^{-12} \mathrm{~Pa}$ range for $\mathrm{Xe}$ were realized.6)
In this paper, other results for $\mathrm{H}_{2}$, and results for $\mathrm{CO}$ and $\mathrm{CO}_{2}$ will be represented.

\section{Measurement System and Method}

The measurement system has already been described in Ref. 6. The second harmonics of a picosecond pulsed YAG laser (Continuum PY61C) was used. Its wavelength and pulse energy are $532 \mathrm{~nm}$ $(2.33 \mathrm{eV})$ and about $30 \mathrm{~mJ} /$ pulse, respectively. The repetition frequency of the laser was $10 \mathrm{~Hz}$ in the present experiments. The laser light was focused into the center of the ionization box of an ion-detection head through a lens with a focal length of $250 \mathrm{~mm}$ and then the view port of the vacuum system. Ions generated in the focused region (ionization space) were led into an electron multiplier (Channeltron) under an applied voltage of $+10 \mathrm{~V}$, and were charge-amplified with a gain of about $10^{6}$ (at $-2.5 \mathrm{kV}$ ).

A preamplifier (Ortec 9305) with a rapid response was combined with the collector electrode of the multiplier. The generated ions produced a series of voltage pulses at the output of the preamplifier. Each pulse corresponds to a single ion. This ion-detection system can produce a pulse with the peak value of a few $100 \mathrm{mV}$ for one incident ion. Therefore, by counting the number of pulses with a pulse counter (SRS SR400), it was possible to determine the number of laser-generated ions precisely.

The distance between the ionization space and the multiplier is about $1 \mathrm{~cm}$. Thus, the time of flight differs with the mass of the ion. Therefore, by setting the gating of the pulse counter at the appropriate time interval, one can selectively count 


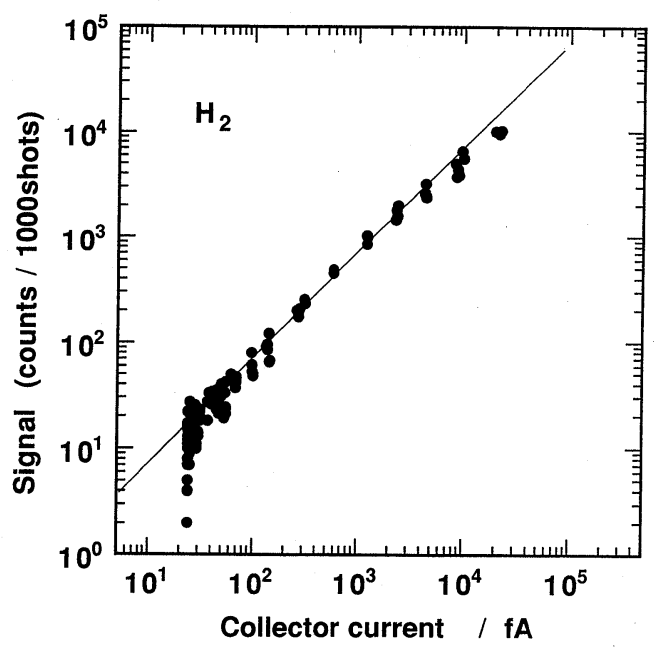

Fig. 1 Pressure measurement by means of an Extractor gauge (horizontal axis) and the nonresonant multiphoton ionization (vertical axis) for $\mathrm{H}_{2}$. The collector current of the Extractor gauge was directly measured by a small-current ammeter.

the number of ions with any mass.

Pressure in the vacuum system was monitored by an Extractor gauge (Leybold IE514). However, we obtained pressure values by directly measuring the collector current of the Extractor gauge with a precise small-current ammeter (Keithley 617).

Sample gases were introduced into the vacuum chamber through two variable leak valves. By keeping the pressure between the two valves at $10^{-1} \mathrm{~Pa}$ to $10^{-5} \mathrm{~Pa}$, it was possible to control the pressure in the XHV range.

\section{Experimental Results and Discussoin}

The vacuum system used can realize lower pressures than the limit of pressure measurement of the Extractor gauge. ${ }^{7)}$ The pressure measurement for $\mathrm{H}_{2}$ gas was carried out at such a low background pressure. Figure 1 shows the result. The horizontal axis represents the collector current of the Extractor gauge. A collector current of $10 \mathrm{fA}\left(1 \times 10^{-14}\right.$ A) corresponds to $1 \times 10^{-10} \mathrm{~Pa}$. The vertical axis represents the count of pulses per 1000 laser shots, which is equal to the number of generated ions.

As seen in Fig. 1, the Extractor gauge shows the measurement limit at about $2.3 \times 10^{-10} \mathrm{~Pa}$, and has no pressure sensitivity below this pressure. On the other hand, the count of pulses continues to vary below this value of pressure; namely the method of pressure measurement by means of this multiphoton ionization with the picosecond laser still has pressure sensitivity at lower pressures than the

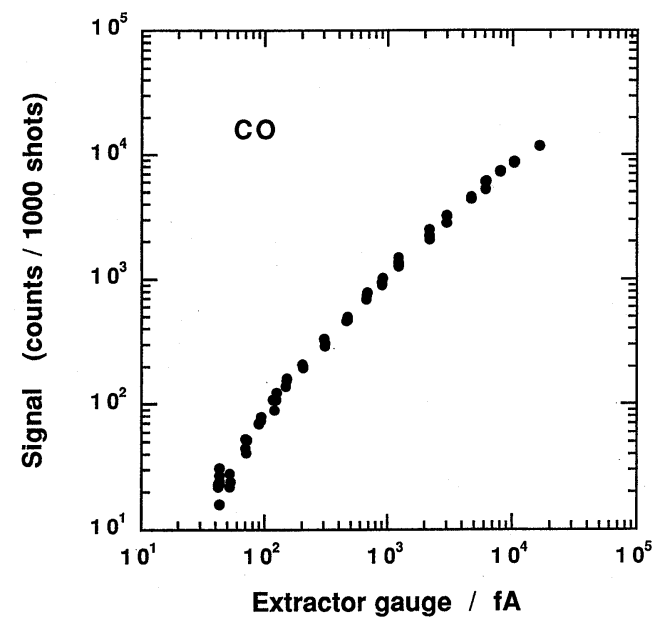

Fig. 2 Pressure measurement for $\mathrm{CO}$ gas. The horizontal axis represents the total pressure measured with the Extractor gauge, and the vertical axis the $\mathrm{CO}$ partial pressure measured by the nonresonant multiphoton ionization.

measurement limit of the Extractor gauge. In Ref. 6 we discussed how this method had a linearity for pressure measurement even at such low pressures. Therefore, Fig. 1 shows that this method of pressure measurement can measure pressures in the range of $10^{-11} \mathrm{~Pa}$. These results are in agreement with the previously reported result. ${ }^{\text {G) }}$

As seen in Fig. 1, at pressures over $1 \times 10^{-8} \mathrm{~Pa}$, the linearity of this method for the pressure measurement begins to degrade. Such a tendency was also observed in the case of other gases. The authors guess that is because, as the number of pulses increases, the pulse counter fails to count them precisely due to the overlapping of pulses.

As decribed in Ref. 6, the combination of the Extractor gauge and the small-current ammeter enables us to measure pressure change on the order of $10^{-11} \mathrm{~Pa}$ at a pressure in the range of $10^{-10} \mathrm{~Pa}$. Introducing $\mathrm{CO}$ gas into the vacuum chamber from the background pressure of $4 \times 10^{-10} \mathrm{~Pa}(40 \mathrm{fA})$, the authors measured the total pressure with the Extractor gauge, and setting the gating of the pulse counter between $2.2 \mu \mathrm{s}$ and $3.2 \mu \mathrm{s}$, the partial pressure was measured by the laser ionization method. The result is shown in Fig. 2. The horizontal axis represents the total pressure (collector current) measured with the combination of the Extractor gauge and the ammeter. The vertical axis represents the count of pulses within the gating time, that is, between $2.2 \mu \mathrm{s}$ and $3.2 \mu \mathrm{s}$, which corresponds to the $\mathrm{CO}$ partial pressure. 


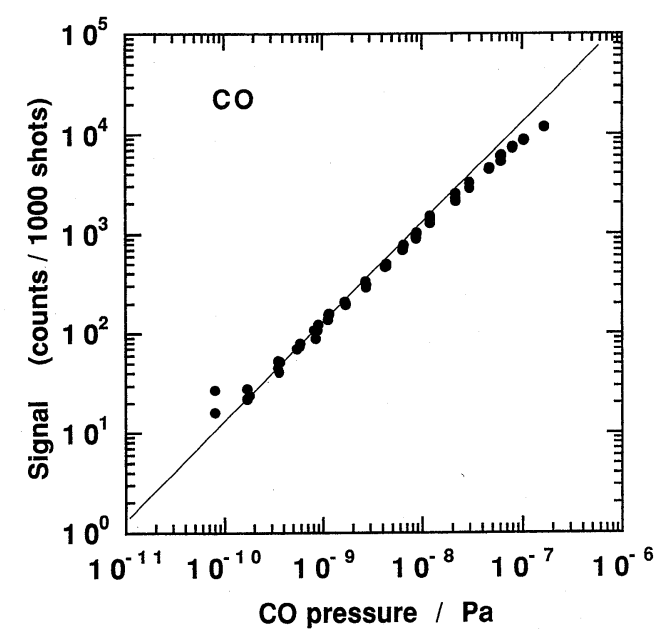

Fig. 3 Pressure measurement for $\mathrm{CO}$ gas. The horizontal axis represents the $\mathrm{CO}$ partial pressure measured by the combination of the Extractor gauge and a small current ammeter.

The CO partial pressure measured with the Extractor gauge can be estimated by subtracting from the measured pressure the background pressure excluding the $\mathrm{CO}$ partial pressure, which can be determined from the ratio of the total count of pulses to the $\mathrm{CO}$ count of pulses measured at the background pressure by the laser ionization method. Figure 3 was obtained in such a fashion. Now the horizontal axis represents the $\mathrm{CO}$ partial pressure obtained by the subtraction (transformed into $\mathrm{Pa}$ representatin with the sensitivity correction) measured with the Extractor gauge.

Figure 4 shows the result for $\mathrm{CO}_{2}$ gas. The horizontal axis represents the $\mathrm{CO}_{2}$ partial pressure measured with the Extractor gauge, which was estimated through the same process as that described above.

As seen in Figs. 1, 3 and 4, the pressure sensitivity for $\mathrm{H}_{2}, \mathrm{CO}$ and $\mathrm{CO}_{2}$ is of the same order of magnitude in this method of pressure measurement. When saturation of ionization is realized for nonresonant multiphoton ionization of some molecules, the pressure sensitivity should be approximately equal for those molecules. Therefore, the result obtained here is reasonable.

\section{Conclusions}

By means of nonresonant multiphoton ionization using a picosecond pulsed YAG laser, pressure measurement for $\mathrm{H}_{2}, \mathrm{CO}$ and $\mathrm{CO}_{2}$ was carried out

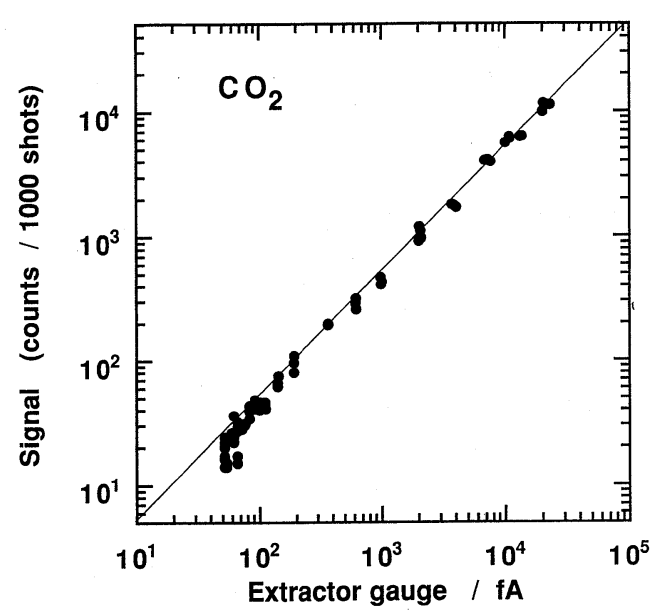

Fig. 4 Pressure measurement for $\mathrm{CO}_{2}$. The horizontal axis and the vertical axis represent the $\mathrm{CO}_{2}$ partial pressure measured by the Extractor gauge and the nonresonant multiphoton ionization, respectively.

in the XHV pressure region. Similarly to the previous results, it was found that the pressure measurement for $\mathrm{H}_{2}$ and $\mathrm{CO}$ could be realized even in the range of $10^{-11} \mathrm{~Pa}$ by this method. Also, the three molecules were found to have pressure sensitivity of the same order of magnitude in this method of pressure measurement.

\section{Acknowledgements}

The authors would like to thank Mr. K. Nakamura and Dr. K. Fukui for their assitance. This work was supported in part by the Special Coordination Funds for Promoting Science and Technology.

\section{References}

1) K. Kokubun, S. Ichimura, H. Shimizu and S. Sekine: Vacuum 44 (1993) 657.

2) K. Kokubun, S. Ichimura, S. Sekine and H. Shimizu: Jpn. J. Appl. Phys. 32 (1993) L1109.

3) S. Sekine, K. Kokubun, S. Ichimura and H. Shimizu: Shinku (J. Vac. Soc. Jpn.) 36 (1993) 323.

4) S. Sekine, K. Kokubun, S. Ichimura and H. Shimizu: Jpn. J. Appl. Phys. 32 (1993) L1284.

5) K. Kokubun, S. Sekine, S. Ichimura and H. Shimizu: Jpn. J. Appl. Phys. 32 (1993) L1368.

6) K. Kokubun, S. Sekine, S. Ichimura, A. Kurokawa and H. Shimizu: submitted to J. Vac. Sci. \& Technol.

7) K. Kokubun, H. Shimizu, S. Ichimura, H. Kawahara and M. Kondo: Shinku (J. Vac. Soc. Jpn) 34 (1991) 14. 